\title{
Del orden social a la cultura digital.
}

\section{From social order to digital culture.}

\author{
Esta obra está bajo una Licencia Creative Commons Atribución 4.0 Internacional.
} DOI: $10.32870 /$ sincronia.axxii.n74.26b18

\author{
Blanca Estela Chávez Blanco \\ Sistema de Universidad Virtual / Universidad de Guadalajara \\ blanca.chavez@redudg.udg.mx / blanca.chavez@suv.udg.mx \\ (MÉXICO)
}

Recibido: 05/03/2018

Revisado: 09/03/2018

Aprobado: $14 / 06 / 2018$

\section{RESUMEN}

La cultura digital está en ebullición, las redes sociales, las aplicaciones móviles, la cartografía virtual del mundo, todo nos remite a unas condiciones espacio - temporales particulares que transforman los modos de habitar el mundo. A través del análisis documental y crítico de la obra de Jean Baudrillard, buscamos establecer en primer lugar los cimientos que estructuran en profundidad dichos modos de habitar, donde las prácticas sociales se alejan cada vez más de las pretensiones de civilidad, organización y racionalidad de otra época. Hiperrealidad, simulación y transparencia son algunas de las nociones que muestran la tendencia actual a negar la distancia como principio de realidad.

Palabras clave: Cultura digital. Mecanismos de disuasión. Simulación. Mundialización. Principio de realidad.

\section{ABSTRACT}

The digital culture is boiling, social networks, mobile applications, the virtual cartography of the world, all refer us to particular spatial-temporal conditions that transform the ways of inhabiting the world. Through the documentary and critical analysis of the work of Jean Baudrillard, we seek to establish in the first place the foundations that structure in depth these ways of inhabiting, where social practices move away more and more from the pretensions of civility, organization and rationality of another time. Hyperreality, simulation and transparency are some of the notions that show the current tendency to deny distance as a reality principle.

Keywords: Digital culture. Deterrence mechanisms. Simulation. Globalization. Reality principle. 


\section{Introducción}

Comprender la cultura que se genera en ambientes virtuales ha sido la preocupación que ha guiado nuestras pesquisas los últimos años, y encontramos en el pensamiento de J. Baudrillard la oportunidad de confrontar nuestras experiencias y reflexiones con una propuesta teórica radical que cuestiona las entrañas mismas de nuestra cultura. Y como es de todos sabido, en la actualidad no se puede dejar de observar la influencia de los desarrollos tecnológicos -particularmente digitales- que trastocan la mayoría, por no decir que todos los ámbitos de la realidad social: economía, política, cultura, arte, educación, gobierno, etc., y el autor fue justamente un crítico de las tendencias que esconden los avances tecnológicos.

Sin embargo, estructurar el pensamiento de Baudrillard no fue sencillo. Sus escritos se caracterizan por una tónica antiacadémica, desestructurada, en resumen, antisistemática. Esto, al tiempo que hace compleja su comprensión, dificulta la posibilidad de "encuadrarlo" en una línea de pensamiento.

Nuestra investigación inicial de orden filosófico antropológico tenía el objetivo, por una parte, de destacar cuál es el papel que los pensadores actuales otorgan al sujeto de conocimiento y por otra esclarecer sus concepciones en torno al ser humano en general (Chávez, 2005). Si alrededor de la idea de hombre giraba la racionalidad moderna ¿en qué soportan los pensadores posmodernos la crítica a dicha racionalidad? ¿en qué fundamentan el rompimiento con la idea de hombre racional? ¿qué propuestas existen para dar respuesta al problema del conocimiento en la actualidad?

Ante tales cuestionamientos, el interés por conceptualizar la idea de hombre en el pensamiento posmoderno nos resultó relevante en tanto que en ella convergen diversas problemáticas de orden epistemológico y antropológico que ayudan a develar la posición que 
toman las corrientes posmodernas. En otras palabras, ante la dificultad que presenta el hecho de que en el pensamiento posmoderno se postule que no existe un paradigma de sustitución de la racionalidad moderna y del hecho de que nos encontremos en la revisión de los postulados modernos, a partir de un fundamento moderno - la concepción de hombre - pretendimos reconstruir y analizar su edificación teórica.

Desde un punto de vista pluridisciplinar sobresalió el pensamiento de J. Baudrillard principalmente porque en su discurso se expresaba una crítica a la racionalidad científica y política de nuestros tiempos, a la vez que problematizaba las condiciones que éstas producen para el ser humano. En este sentido podíamos considerarlo un autor representativo de la posmodernidad.

Baudrillard inició su trabajo intelectual a finales de los sesentas con una crítica sistemática a la postura marxista y a la economía política, expresada principalmente en análisis en torno a los objetos. Entre los libros que reflejan este periodo se encuentran El espejo de la producción, El sistema de los objetos y Crítica a la economía política del signo. Pero existe un segundo momento en su pensamiento, que se caracteriza por su estilo menos sistemático, en el cual aborda problemáticas sociales de la actualidad, sostiene la inconveniencia de postular al valor como eje de nuestra racionalidad, y profundiza en la lógica que se encuentra detrás del orden mundial. Y es precisamente en este cambio en donde pudimos observar que el autor pasa de una concepción del hombre enclavada en la sociología, en donde se vislumbra un ser humano determinado por la organización social, a otra en la que aborda primordialmente al objeto de conocimiento y por ende al sujeto de conocimiento.

Así, el foco de interés del autor fueron los objetos, analizados fundamentalmente desde el orden sociológico. En un primer momento, su análisis se dirigía al papel que los objetos materiales desempeñaban en su relación con los individuos a través el consumo. En un segundo momento, se enfoca en el estatus del objeto de conocimiento en la ciencia. Y precisamente por ello, su discurso lleva un énfasis epistemológico, elaborado a través de la crítica a la relación tradicional entre el sujeto de conocimiento y el objeto, lo cual nos permitió ahondar en el problema del conocimiento, 
aspecto por demás relevante a la hora de visualizar la posibilidad de reconstruir una postura posmoderna en torno al concepto de hombre.

Ahora bien, el pensamiento de Baudrillard se ha expresado en numerosas obras que están atravesadas por el tiempo y que formulan diferentes momentos reflexivos. Esta dificultad, aunada a que el núcleo de su análisis han sido los objetos y no de manera directa y explícita el hombre, determinó que este trabajo fuera ante todo analítico y reconstructivo. Se trataba de extraer y ordenar lo que está implícito y fragmentado en los diversos escritos del autor. Es así que los límites de nuestra investigación obedecieron principalmente a la incapacidad de abordar todas las problemáticas que contempla el autor con la profundidad que requieren, en un discurso que cruza cuatro décadas y al que no se le ha otorgado el tratamiento pormenorizado acostumbrado en el ámbito académico.

En sus lúcidas interpretaciones, posteriores al Sistema de los objetos de la década de los 60 's, el autor expone lo que a nuestro parecer son los mecanismos que hacen perpetuar el orden social. Si bien él plantea el término de mundialización para exponer la cartografía de la realidad social y no el de orden social, este último constructo nos ayudó en la investigación a aglutinar aquello que daba cuenta del ordenamiento y la racionalidad en torno a lo "social", más allá de la dimensión económica.

Dentro de estos mecanismos, el autor expone los chantajes de la seguridad, la búsqueda del control a nivel nuclear y genético, y cómo el sistema de valores y sus relaciones de oposición se desvanecen en la equivalencia, en el intercambio, en la ausencia de referentes, y en el valor centrífugo. A través de términos como el sistema implosivo, la obscenidad, la hiperrealidad y la simulación, exhibe tanto el orden que prima en la actualidad como los valores que lo fundan.

Empero, después de más de quince años de avances tecnológicos, y asumiendo la capacidad de los seres humanos de reconstruir la realidad - incluida la virtual - ¿podemos sostener que la lógica de comprensión de lo social planteada por el autor continua, incluso se exacerba? 
¿podemos sostener que la cultura en la era digital se rige por la simulación que busca eliminar cualquier rasgo de negatividad o de diferencia?

El objetivo de este artículo es entonces poner en relación los resultados de nuestra investigación de la obra de J. Baudrillard con los rasgos de una realidad social mediada por tecnologías en todos sus ámbitos, con la intención de poner a prueba la capacidad explicativa de la perspectiva de Baudrillard en el análisis de la cultura actual.

\section{Mecanismos de disuasión}

Vivimos ni más ni menos la realización de la utopía, es decir, su hundimiento en lo real.

Jean Baudrillard (1984, p.64)

En Las estrategias fatales el autor presenta esta frase criptica que refleja su crítica fundamental al orden social contemporáneo, la falta de creencia en lo indeterminado por una consumación más o menos real. Un orden social en donde el manejo del código prima en el ámbito económico, en donde la transparencia oscurece el espacio político, y donde la masa es reducida a su nivel más bajo, el victimario, en el ámbito cultural. Y como contexto, la mundialización, término que sintetiza el proceso de control de los intercambios a través de las técnicas, del mercado y de la información.

La fuerza del orden social se encuentra entonces en el control de la información y de las técnicas que reconstruyen y acumulan los datos que emergen del mundo hasta en sus más mínimas fracciones, creando una saturación de información sin precedentes. Es en este sentido en el que J. Baudrillard afirma que, a través de la mundialización, se realiza la escritura automática del mundo, en donde ya no hay espacio para crear la realidad, ese ámbito que históricamente ha permitido interpretar el mundo.

Su perspectiva refiere un nuevo orden mundial convertido en un sistema integrista de lo vacío, porque no se dirige a la consolidación de un ideal social, o a conciliar las diferencias, sino a la 
integración desprovista de sentido, sustentada en mecanismos de disuasión. El autor lo explica en comparación con la intencionalidad moderna, donde se promulgaba el respeto y la búsqueda de integración de la diferencia, se defendían los derechos humanos como soporte del sistema de valores de la sociedad; sin embargo, en la actualidad, aunque se sigue proclamando el mismo discurso, en la práctica se produce la anulación de esta universalidad de los valores en pro de la mundialización, que hace tabla rasa de todas las diferencias y de todos los valores, inaugurando lo que él llama una (in) cultura perfectamente indiferente. Porque como él explica, cuando lo universal desaparece, ya no queda sino la omnipotente tecnoestructura mundial frente a las singularidades que vuelven a ser salvajes y quedan a merced de sí mismas.

En este contexto, para J. Baudrillard "lo anomálico ya no es lo que está al margen, en desequilibrio, en déficit orgánico, sino lo que resulta del exceso de organización, de regulación y de racionalización de un sistema" (2000, p.114). Al modo en cómo se desarrolla el cáncer, en el cual existe una producción excesiva de células que ya no son producto de un agente extraño con el cuál el cuerpo puede pelear, sino que son las propias células del cuerpo las que se reproducen inconteniblemente, llegando a una producción excesiva de lo positivo. En este sentido es que postula que, "las sociedades en apariencia todopoderosas se desestabilizan desde el interior [...] cuanto más quisiera el sistema reabsorber las anomalías, más entrará en la lógica de la superorganización y más alimentará su crecimiento excéntrico" (2000, p.114), donde lo urgente entonces es liberarse de esta visión racionalista o super-racionalista, porque no se puede pensar en acelerar o corregir el sistema, éste seguirá creciendo bajo la misma lógica. De ahí la visión apocalíptica criticada al autor ${ }^{1}$.

Desde una perspectiva estética, y un tanto más actualizada respecto a los avances tecnológicos, Han Byung-Chul en su libro la salvación de lo bello explica cómo la mundialización

\footnotetext{
${ }^{1}$ Cf. Caroline Bayard, Épistemologie du corps et postmodernité: de l'apocalypse de Baudrillard à l'Einfühlung de Maffesoli, Sociologies et sociétés, vol. XXIV, n¹, printemps 1992.
} 
expulsa aquello que resulta opaco, extraño, aquello que requiere un alejamiento interpretativo, y privilegia justo aquello que no se nos opone, aquello que nos resulta transparente,

Lo pulido, pulcro, liso e impecable es la seña de identidad de la época actual. Es en lo que coinciden las esculturas de Jeff Koons, los iPhone y la depilación brasileña. ¿Por qué lo pulido nos resulta hoy hermoso? Más allá de su efecto estético, refleja un imperativo social general: encarna la actual sociedad positiva. Lo pulido e impecable no daña. Tampoco ofrece ninguna resistencia. Sonsaca los «me gusta». El objeto pulido anula lo que tiene de algo puesto enfrente. Toda negatividad resulta eliminada. $(2015$, p.3).

La sociedad positiva tiende pues a la eliminación de lo distinto, a la reproducción de lo mismo, de sí mismo. Y en este sentido para este último autor, el arte de Jeff Koons como baluarte de la cultura actual "ejerce una sacralización de lo pulido e impecable. Él escenifica una religión de lo pulido, de lo banal; es más una religión del consumo, al precio de que toda negatividad debe quedar eliminada" (2015, p.5).

Volviendo a la argumentación de J. Baudrillard sobre el orden mundial, reconocemos entonces que los detonantes que lo justifican son principalmente la amenaza de la muerte y la inseguridad, por lo que transitamos del dispositivo panóptico de vigilancia ${ }^{2}$ hacia la disuasión, en donde el sistema de gobierno es poderoso en tanto genera desilusión e incertidumbre colectiva, como una forma de desestabilizar el cuerpo social, principalmente en el ámbito económico, con las fluctuaciones de las tasas de interés, de la bolsa de valores y de los intercambios, claro desde un contexto europeo. Si nos inclinamos a territorios latinoamericanos las desestabilizaciones vendrían además del crimen organizado, de la corrupción gubernamental, y de la ingobernabilidad que ocurre en lugares como México. Éste sería pues el estadio actual de la relación social, en la que ya no existe ni perspectiva ni persuasión sino disuasión.

\footnotetext{
${ }^{2}$ El término de dispositivo panóptico de vigilancia hace referencia a lo que Michel Foucault concibe como un mecanismo de dominación propio de la sociedad disciplinaria en el que se articulan diversas estrategias de observación y control de las conductas de los individuos.
} 
De manera concreta, para el autor los mecanismos que mantienen el control social tras el modelo de la disuasión son: la seguridad y el manejo de lo nuclear - genético. En primer lugar, como ya lo mencionamos, la disuasión se plantea bajo la manifestación de la necesidad de seguridad mundial en pro del bienestar de la sociedad, se disuade a los sujetos de tener cualquier intervención social. Y no es que la seguridad sea un hecho en la sociedad, el terrorismo es una clara muestra de que no es así, es en todo caso la exhortación a la búsqueda de seguridad el mecanismo que permite desarticular las relaciones sociales gracias al discurso del peligro y la incertidumbre permanente. Para J. Baudrillard, la deuda mundial por ejemplo supera los fondos disponibles, igual que los armamentos nucleares superan la destrucción del planeta: a lo único que conducen estos mecanismos es a someter a los humanos al destino del crédito, de la amenaza y de la disuasión. Así, resulta "un proceso contradictorio, pues la información bajo todas sus formas, la seguridad bajo todas sus formas, en lugar de intensificar o de crear la "relación social», son al contrario unos procesos entrópicos, unas modalidades del fin de lo social" (Baudrillard, 2002, p.132). Del mismo modo, si bien las redes sociales en la actualidad intensifican los intercambios comunicativos, esto no implica necesariamente que las relaciones sociales se fortifiquen.

Pensemos en el contexto actual, los problemas de inseguridad por redes de pornografía infantil, trata de personas, secuestros, ciberbullying, muchos de ellos generados a través de información que se publica en las redes sociales, son fenómenos que empujan a la disuasión, sin embargo, pareciera que no es más el poder institucional o gubernamental quien orquesta la disuasión con miras a mantener el control por lo complejo de sus mecanismos de vigilancia, ya que es justo la ingobernabilidad que vivimos particularmente en México en un estado de guerra el que disuade permanentemente a la población a organizarse.

El segundo mecanismo de disuasión es el manejo de lo nuclear y lo genético, en el que se busca manipular lo contingente, controlar a la vez "trayectoria, energía, cálculo, fisiología, psicología, entorno - nada puede ser abandonado a la contingencia, se trata del universo total de la norma - ahí la ley ya no existe, es la inmanencia operativa de todos los detalles la que legisla" 
(Baudrillard, 2002, p.68). De tal forma, el espacio que regía la convivencia humana ha dado paso a un control superior, en el que se regula la vida de los sujetos, al grado de que se pretende convertirla en un universo cerrado e integrado en el que todo sea transparente. En estos términos, tanto lo nuclear como lo genético impiden el contacto y la realización de la realidad y cualquier búsqueda de sentido en ésta.

En otro nivel, pero bajo la misma lógica, pensemos en la actualidad cómo la tendencia a prevenir virtualmente las contingencias nos lleva a intentar controlar -al menos informativamentetodos nuestros desplazamientos a través de aplicaciones (Uber, Google Maps, Kayak, etc.), o cómo nuestro actuar está mediado invariablemente por la mirada digital de la realidad: la necesidad de contar con la información del tiempo, de eventos, de comercios, de problemas de tránsito, de todo aquello que nos permita "prevenir" nuestra movilidad; sólo por hablar de un tema.

Las interpretaciones de Baudrillard, de corte europeo, pareciera que sólo son aplicables como tendencias generales, pensando en la realidad particularmente mexicana, siendo además una forma degradada de éstas. Contra el chantaje de la seguridad se impone una inseguridad obscena, contra la búsqueda del control nuclear y genético se impone el control informacional.

La disuasión podemos entenderla entonces no sólo como un mecanismo particular de los gobiernos por "controlar" la movilización social a través de los chantajes de la seguridad, sino también como la invitación culturalmente obligada a participar en una forma de imponernos al mundo que nos lleva a alejarnos permanentemente de éste, a negar cualquier rasgo de contingencia, principalmente por los medios con que contamos para despedazarlo. En términos de Byung-Chul, "lo bello mismo resulta satinado cuando se le quita toda negatividad, toda forma de conmoción y vulneración. Lo bello se agota en el «me gusta». La estatización demuestra ser una «anestetización»". (2015, p. 5).

\section{La simulación}


Así, el proceso de disuasión impone una racionalidad donde la omisión de lo negativo prima, pero en una relación donde uno de los polos se suprime se acaba por eliminar también las reglas del juego mismo que se construían por la oposición entre sus elementos. De acuerdo con J. Baudrillard, la trasformación comenzó con el capital, que fue el primero que se alimentó a lo largo de la historia "de la desestructuración de todo referente, de todo fin humano, quien primero rompió todas las distinciones ideales entre lo verdadero y lo falso, el bien y el mal, para sentar una ley radical de equivalencias y de intercambios" (Baudrillard, 2002, p. 52). Al romperse la relación entre los polos (positivo-negativo, bueno-malo, bello-feo, valioso-insignificante) se ha sumido a los valores en la indiferenciación. Tal cuestión se ve reflejada en la información y la comunicación, cuyo principio es "el de un valor ya no referencial, sino fundado en la circulación pura [...] es una definición del valor totalmente nueva, un valor puramente centrífugo." (Baudrillard, 2000, p.40).

Y la secuela es que al no distinguir lo verdadero de lo falso, el bien del mal, lo bello de lo feo, lo que tiene valor de lo que no lo tiene, la tendencia es almacenar todo, lo cual produce una devaluación sin precedentes. Sólo cuando existe una escena, imaginación o ilusión es cuando realmente atribuimos sentido a algo, la dificultad está en que en la actualidad lo que tenemos es una sobreexposición del mundo (principalmente a través de los modelos de simulación y de la producción-circulación que hacen los medios de comunicación). En palabras de J. Baudrillard "la obscenidad es hoy, por el contrario, la de la superrepresentación. Nuestra obscenidad radical ya no es la de lo oculto y de lo inhibido, sino la de la transparencia de lo social" (1984, p.67).

En este mismo sentido, los polos que permitían dimensionar la realidad - causa-efecto - son absorbidos el uno en el otro, por lo que en los diferentes ámbitos (político, económico, científico, etc.) lo que existe son descripciones fragmentadas, modelos analógicos creados para hacer inteligible el mundo. A diferencia del sistema explosivo de Occidente donde se tendía a la expansión dirigida y a la universalización de la ley ahora nos encontramos ante el fracaso de dicho sistema en pro de uno implosivo, y con él inicia la simulación que se manifiesta en una suplantación de lo real por los signos de lo real, en un mecanismo de disuasión en el que el proceso real tiene su doble 
operativo. "Esa es la forma ideal de la simulación: derrumbamiento de los polos, circulación orbital de los modelos (es también la matriz de todo proceso implosivo)." (Baudrillard, 2002, p.128).

De esta forma, la simulación crea lo hiperreal: "la generación por los modelos de algo real sin origen ni realidad" (Baudrillard, 2002, p.9), que se produce por medio de análisis basados en la miniaturización, es decir, en la reproducción de lo real desde sus mínimos componentes a través de modelos a semejanza. El resultado, es un "mundo completamente recensado, analizado y luego resucitado artificialmente disfrazándolo de realidad $^{3}$, en un mundo [...] de alucinación de la verdad, de chantaje a lo real, de asesinato de toda forma simbólica" (Baudrillard, 2002, p.23). Estamos sumergidos en un orden que ya no nos pertenece, que es al mismo tiempo la realización del pensamiento del ser humano y su automatización, pero que por lo mismo nos ha superado en cuanto a las posibilidades que tenemos de oponernos a él.

En correspondencia, a nivel social, la masa pierde su estatus de sujeto, no representa, simula. Es la simulación de las estadísticas, de los sondeos, sin referente preciso, es ahí donde radica el fin de lo social.

Tenemos así la cuestión de hallarnos en una lógica de la simulación que no obedece a la lógica de los hechos. Por un lado, existía la concepción de historia que suponía una continuidad, partía de un principio de explicación, pero esta idea ha perdido su fuerza gracias a los medios con que contamos para representar la historia, la hacemos cada vez más visible, presente en tiempo real - virtual -, y, por tanto, desprovista de sentido alguno. Por otro lado, está el acontecimiento, el cual no parte de principio alguno de explicación, incluso puede romper las racionalizaciones históricas o políticas, de ahí su importancia. Sin embargo, tanto la historia como el acontecimiento se desvanecen como tales, la primera ha perdido su definición y su sentido como acontecimiento; el segundo, por su parte, se aleja de nosotros a través de todos los medios que tenemos para

\footnotetext{
${ }^{3}$ El resaltado es del traductor.
} 
recordarlo. Es decir, el acontecimiento, la fuerza de los hechos, se disipa en medio de la indiferencia que produce el exceso de información, de datos, de registros, incluso, por la saturación de sentido.

De igual forma, en el ámbito especializado de los medios de comunicación se genera un mecanismo de circulación donde el manejo y la producción de la información anulan la relación entre lo verdadero y lo falso, al basarse exclusivamente en la credibilidad instantánea, eliminando cualquier criterio de referencia. Lo que importa no es la verdad o la falsedad, sino que la información sea creíble al menos por un período de tiempo. Asimismo, autodestruye la información como acontecimiento, por el mismo mecanismo en el que produce y presenta la información, se vuelve incierta.

En medio de este contexto de reconstrucción simulada del mundo, se presenta lo virtual, que para J. Baudrillard pone fin tanto a la realidad como a la imaginación de lo real gracias al manejo de la información en tiempo real, lo cual imposibilita la imaginación del pasado o del futuro dentro de una sincronía de todos los lugares y tiempos en la virtualidad intemporal. Aquí es donde el autor prefigura el triunfo del tiempo real sobre el pensamiento crítico o histórico, sobre cualquier articulación lógica de la realidad (2000, p.127).

El tiempo real no contempla la estructuración pasado, presente, futuro, ni la causa-efecto, categorías que permitían conformar la realidad. Ahora queda la indiferencia ante la memoria, que responde a los excesos por objetivar la historia, por reconstruirla hasta en sus más mínimos detalles, por presentarla en tiempo real.

En síntesis, con la simulación como circulación de los modelos sin origen ni realidad, como simulación virtual que comprime la información en un tiempo presente, nos vemos confrontados a una realidad ajena a la lógica que parecía articular nuestra comprensión del mundo; y aunque parece un seudo problema ya que históricamente hemos cambiado los modos en que comprendemos el mundo, lo inquietante para el autor es la pérdida de referencia, la circulación indiferenciada de las cosas y de los valores, que dificulta la contrastación o el diálogo a nivel comunicativo. 
Si bien hasta el momento el discurso del autor nos deja en la indefensión, sin posibilidades de contrarrestar el curso del orden social, no es muy diferente a la interpretación de Gilles Lipovetsky con La era del vacío (1986), Giovanni Sartori con Homo videns (1998), Los bárbaros de Alejandro Baricco (2006), o La sociedad de la transparencia de Han Byung-Chul (2013). Lo relevante del análisis de J. Baudrillard radica en la potencialidad que tiene para desnudar la supuesta transparencia de la cuál somos usuarios involuntarios.

\section{La transparencia}

La transparencia es un valor que toma fuerza en la actualidad, legitimándose en oposición a los llamados sistemas ideológicos del siglo pasado, o a estados totalitarios en el ámbito político, en oposición también a la corrupción en el ámbito económico, o a la manipulación mediática en el ámbito de la comunicación. En los hechos, la transparencia se evidencia con la propuesta de software libre donde cualquiera puede usar, estudiar, distribuir o mejorar al desarrollo tecnológico de una plataforma como Moodle; o en proposiciones como la de Wikipedia, que muestra las discusiones y construcciones discursivas de la comunidad en torno a un tema. Transparencia también de las relaciones sociales que dejan huella en cada red social que registra los amores, desamores y amistades. Transparencia del espacio privado que presenta la recreación audiovisual y fotográfica de los viajes y eventos importantes. Es la transparencia que evidencia tanto el procedimiento detrás de la gestión, de la creación, o de la construcción, como la forma en que transcurren los eventos.

En este sentido, Byung-Chul explica en su libro La sociedad de la transparencia que: "Las cosas se hacen transparentes cuando abandonan cualquier negatividad, cuando se alisan y allanan, cuando se insertan sin resistencia en el torrente liso del capital, la comunicación y la información" (2013, p.11). Es la transparencia virtual y obscena que expone el mundo hasta en sus más mínimos detalles. 
La contraparte es que la transparencia se sostiene en el intento de operacionalizar el mundo, de someter al cálculo y al control todo fenómeno, incluso se reduce al ser humano "hasta convertirlo en un elemento funcional de un sistema. Ahí está la violencia de la transparencia." (Byung-Chul, 2013, p.14). Es así que para el autor la sociedad de la transparencia es "un infierno de lo igual".

Si pensamos por ejemplo en el fenómeno de los Youtubers convertidos en Influencers, ganando cifras millonarias por el número de seguidores que tienen, que se transforman en líderes de opinión no por su erudición, formación, preparación o por la credibilidad que tengan sobre un tema sino por la capacidad de llamar la atención de un público que cree y confía más en una persona ordinaria, podemos observar esta tendencia a congeniar con aquellas propuestas positivas que no presenten recovecos, dobleces o matices. Siendo lo más recurrido Gameplay, humor o "cosas diferentes", presentaciones vacías y repetitivas que determinan el interés de la gente por los Youtubers, en donde el valor del esfuerzo - trabajo es sustituido por aquel de la espontaneidad y la repetición. O el fenómeno de los Memes que son reproducidos al infinito y generan una "opinión" generalizada y burlesca respecto a la realidad, sin la menor intención de fundamentar o documentar la crítica. Son como cápsulas informativas en formato cómico que permiten compartir una catarsis social. De nuevo el infierno de lo igual.

Es así que la transparencia tanto como la simulación terminan soportando una tendencia al cálculo, al control a través de la reconstrucción operativa del mundo. En tanto los medios con que contamos culturalmente para comunicarnos, para socializar, para reconocernos en otros generan la reproducción al infinito de lo intrascendente, presentado en llamaradas intermitentes que mantienen el interés activo.

\section{Conclusiones}

Los mecanismos de disuasión planteados a través del pensamiento de J. Baudrillard nos hacen reflexionar en una perspectiva a la que de una manera deliberada nos resistimos, lo que en otros 
tiempos implicaba analizar "el poder". Sin embargo, dejar de pensar en el poder centralizado en el gobierno o en el capital, o rechazar las explicaciones basadas en conspiraciones no implica que no sea necesaria la comprensión de las lógicas que gobiernan nuestra vida cultural. Es decir, creer en la capacidad de reconstrucción cultural de la realidad no nos impide pensar en nuestros límites compartidos.

En este sentido, J. Baudrillard aporta una perspectiva muy pertinente para comprender dichos límites. A través del término simulación, que da cuenta de la duplicidad que genera la digitalización del mundo y donde ésta supera la lógica donde primaba el principio de realidad, el autor expone la tendencia a negar la distancia como principio de la cultura actual, la distancia interpretativa del arte, la distancia simbólica del mundo, la distancia comunicativa con los otros. Sea por la escritura automática del mundo o por la obscenidad de la transparencia: "la exhaustiva visibilidad del objeto destruye también la mirada" (Byung-Chul, 2015, p.5).

En nuestra tesis doctoral en sociología L'e-rationalité postmoderne. Étude de l'être ensemble électronique (la e-racionalidad postmoderna. Estudio del ser juntos electrónico) hicimos un análisis de experiencias en la red social Facebook donde reconocimos justamente que las tendencias societales se dirigían a la búsqueda de los otros, a perderse en un espacio compartido y a esperar... esperar a que llegue el momento perfecto en que "algo" nos despierte del letargo de lo mismo. Sin embargo, ese "algo" no tenía cualidades diferenciales, no los interpelaba, no los hacía inquietarse en lo más mínimo - cualidades propias de aquello que nos resulta "diferente", extraño - no, en todo caso implicaba un sobresalto de lo mismo, una "oportunidad" para encontrarnos con otros que compartieran el mismo momento emocional, para estar conectado e informado sobre aquello que todos sabían en ese momento dado (Chávez, 2017).

Continuar con la tendencia a reproducir los gustos, las ideas, las opiniones y a buscar exclusivamente aquello que se comparte pareciera la forma cultural de manifestar el acuerdo con un mundo simulado, donde se mantiene todo a distancia, y donde se puede borrar del paisaje aquello que perturba. Si bien existen fenómenos dignos de reconocer en las redes sociales, la 
tendencia en el uso de espacios virtuales es efectivamente la repetición, la búsqueda de afectividad, aspectos que exacerban lo que Byung-Chul llama la sociedad positiva.

\section{Referencias:}

Baricco, A. (2006). Los bárbaros. Ensayo sobre la mutación. Barcelona: Anagrama. Traducción. Xavier González Rovira. 2008. Titulo original. I barbari, Fandango Libri, Roma.

Baudrillard, J. (1984). Las estrategias fatales. Barcelona: Anagrama.

Baudrillard, J. (2000), Pantalla total. Barcelona: Anagrama.

Baudrillard, J. (2002), Cultura y simulacro. Barcelona: Kairós.

Byung-Chul, H. (2013), La sociedad de la transparencia. Barcelona: Herder. [Traducción. Raúl Gabás, 2012]. Título original Transparenzgessellschaft. Berlín: MSB Matthes \& Seitz Verlag.

Byung-Chul, H. (2015), La salvación de lo bello. Barcelona: Herder. Traducción. Alberto Ciria. Título original: Die Errettung des Schönen.

Chávez, B. (2005). La posmodernidad según Jean Baudrillard. Ensayo de reconstrucción de su concepción antropológica (Tesis de maestría). Departamento de Filosofía, Universidad de Guadalajara, Guadalajara, México.

Chávez, B. (2017). L'e-rationalité postmoderne. Étude de l'être ensemble électronique (Ph.D. Disertación Doctoral). L'école doctoral « Sciences humaines et sociales : cultures, individus, sociétés » (ED 180). Université Paris Descartes, Paris Francia.

Lipovetsky, G. (1986), La era del vacío. Ensayos sobre el individualismo contemporáneo. Barcelona: Anagrama.

Sartori, G. (1998), Homo videns. México: Alfaguara. 